\title{
ON TETRAD ANALYSES APPARENTLY INCONSISTENT WITH MENDELIAN LAW
}

\author{
ØJVIND WINGE and CATHERINE ROBERTS \\ Carisberg Laboratory, Copenhagen
}

Received 24.vi.54

\section{INTRODUCTION}

AT the Seventh International Botanical Congress in Stockholm in 1950, Professor I. E. Glushchenko (Russia) presented a paper which gave rise to a lively discussion of mendelian segregation, whose universal validity and dependence upon a chromosome mechanism was not admitted by the Russian investigator. On this occasion Professor A. Quintanilha (Portugal) asked Glushchenko how he would explain, other than by a chromosome mechanism, the simple $2: 2$ segregations which result from tetrad analyses of various monohybrid Cryptogams such as Chlamydomonas, Coprinus, Neurospora and Saccharomyces. Glushchenko replied that he was not familiar with these organisms but assumed that the results were due to a statistical phenomenon and that other numerical ratios besides the $2: 2$ should occur. To this statement Quintanilha replied that it was not a question of statistics, but rather of a fundamental principle, according to which every ascus or basidium of a monohybrid yields a segregation in the ratio of $2: 2$.

As is well known, Hans Winkler in I930 put forward his conversion theory, according to which a gene in a heterozygote may be converted into the allemorphic gene, so that a dominant gene may be changed into a recessive and vice versa. Also, according to this theory, a whole group of linked genes may be transformed at one step into a corresponding group of allelomorphs. Furthermore, the frequency of conversion of a dominant gene in the recessive direction need not always be the same as that of a recessive gene in the dominant direction. Winkler's principal aim was to show that T. H. Morgan's theory of crossing over need not be correct, since gene conversion could also explain those phenomena normally explainable on the basis of crossing over. Winkler did not, however, deny the position of genes along the length of the chromosome.

An important part of Winkler's argument was the fact that several investigators had found that tetrad analyses of certain Cryptogams sometimes yielded segregation ratios deviating from the expected $2: 2$ (Fr. Wettstein in Funaria hygrometrica, Brunswick in Coprinus fimetarius, Kniep in Aleurodiscus polygonius, Hanna in Ustilago zeae, etc.)-exceptions which could be accounted for on the basis of gene conversion.

In general, Winkler's theory received little support until Lindegren and his associates (Lindegren, 1949, 1953; Mundkur, 1949), on the basis of frequent deviations from the expected $2: 2$ ratios in the 
tetrad analyses of yeasts (Saccharomyces), subscribed to it with much enthusiasm. They found quite commonly 3 dominant : I recessive segregations or $4 \mathrm{~d}: \mathrm{or}, \mathrm{I} \mathrm{d} \mathrm{:3r}$, and o d : $4 \mathrm{r}$ and were thereby of the opinion that they had given the death blow to mendelism, or, at least, that mendelian theory needed a profound modification.

In what follows we will disregard the numerous sources of error which should be considered in tetrad analyses: (I) overlapping of generations, which carries with it a grave risk in the study of yeast genetics, (2) mutation, which here just as in all other organisms can lead to deviations from the expected segregation, (3) adaptation to the ability to ferment certain sugars or to grow in the absence of certain growth substances, which can lead to analytical difficulties, (4) cytoplasmic inheritance of certain respiratory enzymes, which has been shown through the work of Ephrussi (1953) and his associates to exist in yeasts, and (5) polyploidy, which could easily lead to abnormal segregation, although its occurrence in yeasts has not yet been established with certainty.

After not having found during the course of several years in our yeast genetic investigations any deviations from mendelian segregation which could not be explained on the basis of mutation, we observed (Winge and Roberts, I950a) in a monohybrid heterozygous for maltose fermentation, $M m$, a single 4 -spored ascus (among I 3 ) which yielded a $3 M$ : I $m$ segregation. As a working hypothesis, we assumed that an extra mitosis had taken place in the young 4-nucleate ascus so that $4 M$ - and $4 m$-nuclei were produced, but that only 4 uninucleate spores were formed and that these, by chance, contained $3 M$-nuclei and I $m$-nucleus while the fourth $M$-nucleus and the 3 remaining $m$-nuclei were not incorporated in any spore. In support of this hypothesis we stated that both $5^{-}$and 6-spored asci had been observed, showing that the occurrence of extra mitoses was actually demonstrable in this material. Genetic analyses of these asci with more than 4 spores showed that in a single ascus either maltose fermenters or non-fermenters could be in excess.

This explanation was rejected by Mundkur (1950) who showed that the deviating segregation ratios in his own material, which originated from Lindegren and which contained a series of genes in the heterozygous condition, were not explainable on the basis of our hypothesis. He correctly argued that even if an ascus becomes 8-nucleate it could never contain more than 4 biotypes, and that to adopt this hypothesis for explaining his results would require the further assumption that more than 4 biotypes would have to be present among the assumed 8 nuclei in the ascus. It should be noted that we had never maintained that our hypothesis could explain all exceptions to the expected $2: 2$ ratios in tetrad analyses. As it will appear from the following, however, our hypothesis of the significance of extra mitoses actually contained the correct idea but lacked an important corrective. 


\section{GENETIC ANALYSIS OF DEVIATING ASCI IN OUR MATERIAL.}

In material originating from a hybridisation between Saccharomyces cerevisiae, type Yeast Foam and $S$. chevalieri we obtained, following various spore crossings, a hybrid (230) which was heterozygous in two gene pairs, $M_{3} m_{3} R_{2} r_{2}$-i.e. it was heterozygous with respect to one of the four maltose fermentation genes previously isolated and to one of three raffinose fermentation genes previously isolated (Winge and Roberts, 1948, I950b, I952 ; Gilliland, 1949). This hybrid yielded out of a total of 37 tetrads, 6 which gave ratios other than

TABLE 1

Abnormally segregating asci from hybrids 230, 254 and 236 all $\mathrm{Mm} \mathrm{Rr}$

\begin{tabular}{|c|c|c|c|c|c|c|}
\hline Hybrid & $\begin{array}{l}\text { No. of } \\
\text { asci }\end{array}$ & $M+R$ & $M+r$ & $m+R$ & $m+r$ & \\
\hline 230 & $\begin{array}{l}1 \\
1 \\
2 \\
1 \\
I\end{array}$ & $\begin{array}{l}\cdots \\
1 \\
2 \\
2 \\
I\end{array}$ & $\begin{array}{l}2 \\
1 \\
\cdots \\
\cdots \\
2\end{array}$ & $\begin{array}{l}1 \\
2 \\
1 \\
2 \\
1\end{array}$ & $\begin{array}{c}\mathbf{1} \\
\cdots \\
\mathbf{I} \\
\cdots \\
\cdots\end{array}$ & $\begin{array}{l}=1 R: 3 r \\
=3 R: 1 r \\
=3 R: 1 r \\
=4 R: 0 r \\
=3 M: 1 \mathrm{~m}\end{array}$ \\
\hline 254 & $\begin{array}{l}1 \\
3\end{array}$ & $\begin{array}{l}1 \\
2\end{array}$ & $\begin{array}{l}1 \\
I\end{array}$ & $\begin{array}{c}\cdots \\
\mathbf{I}\end{array}$ & $\begin{array}{l}2 \\
\cdots\end{array}$ & 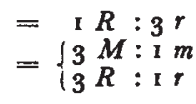 \\
\hline 236 & $\begin{array}{l}\text { I } \\
\text { I }\end{array}$ & $\begin{array}{l}2 \\
1\end{array}$ & $\begin{array}{l}2 \\
1\end{array}$ & $\ddot{2}$ & $\begin{array}{l}\cdots \\
\cdots\end{array}$ & $\begin{array}{l}=4 M: 0 m \\
=3 R: 1 r\end{array}$ \\
\hline
\end{tabular}

$2: 2$; these comprised $3 M:$ I $m$ (I ascus), $3 R:$ I $r$ (3 asci), I $R: 3 r$ (I ascus), and $4 R: 0 r$ ( $\mathrm{I}$ ascus). The last-named ascus was undoubtedly the result of overlapping of generations.

Another hybrid (254), having the same origin and genetic formula as $\mathrm{H} 230, M_{3} m_{3} R_{2} r_{2}$, yielded deviating ratios also. We analysed I07 tetrads from $\mathrm{H}$ 254, of which I03 segregated out normally ( $2 M: 2 m$ and $2 R: 2 r$ ), while the four irregular asci comprised 3 cases of $3 M:$ I $m+3 R:$ I $r$ and one I $R: 3 r$.

Finally, a third doubly heterozygous hybrid (236) was produced through spore crossing. It originated from $\mathrm{H} 230$ and had exactly the same formula, $M_{3} m_{3} R_{2} r_{2}$. In addition to I 7 normally segregating asci (2 $M: 2 m+2 R: 2 r)$ there were two deviating tetrads, one which gave a $4 M: 0 m+2 R: 2 r$ segregation, and one which gave a $3 R:$ I $r+2 M: 2 m$ segregation.

We thus had ample material to investigate in the hope of explaining the occurrence of these abnormally segregating asci, which are shown in table $\mathrm{I}$.

These I2 deviating asci constituted $7 \cdot 4$ per cent. of the total number of asci analysed, of which I5 $^{\mathrm{I}}$ segregated normally. In 
order to determine the cause of these deviations we undertook both genetical and cytological investigations of the material. A single case will serve to illustrate the results of the genetic investigations.

In table $\mathrm{I}$ the second deviating ascus of $\mathrm{H} 230$ contained 4 spores (Nos. 25, 26, 27 and 28) which gave rise to 4 single-spore cultures which, on the basis of their fermentative ability, had the genetic formulas

$$
M+R \quad M+r \quad m+R \quad \text { and } m+R
$$

and consequently this tetrad segregated out as $2 M: 2 m+3 R:$ I $r$.

Since all of our yeast types contain the gene $D$ for self-diploidisation (Winge and Roberts, 1949), it was to be expected that all spores isolated would give rise to homozygous single-spore cultures. We obtained, however, by isolating spores from each of the four diploid single-spore cultures of this deviating tetrad and analysing the subsequent cultures the following segregation ratios with respect to maltose and raffinose fermentative ability :

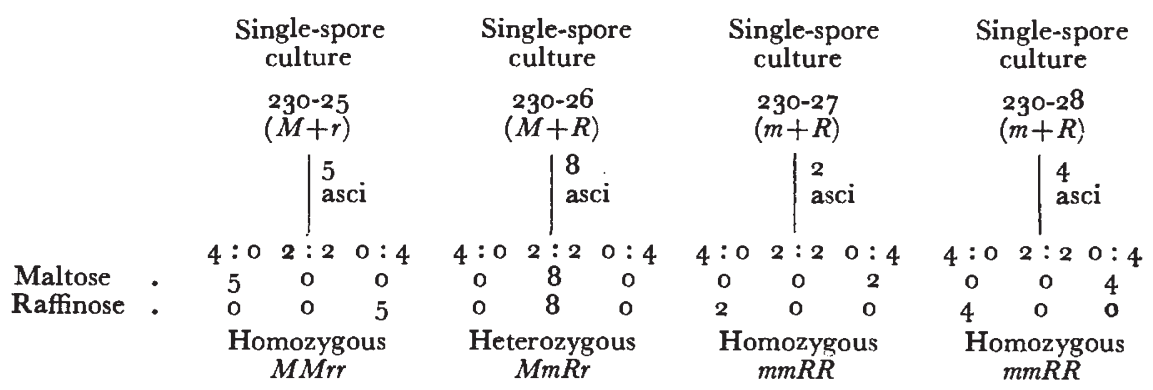

It is seen that three of the four single-spore cultures were homozygous, $M M r r$, $m m R R$, and $m m R R$, while the fourth (230-26) was heterozygous in both gene pairs, $M m R r$.

Since this deviating ascus, $230-25$ to 28 , undoubtedly should have given a dicratic segregation of the type $2 M r: 2 m R$, it is evident that it is one of the $M r$-spores which was abnormal, in that this spore gave rise to a culture able to ferment both maltose and raffinose. It can be concluded that an extra $m R$-nucleus was incorporated in the spore together with the theoretically expected $M r$-nucleus, and this is not difficult to explain : an extra mitosis occurred in the ascus, and the abnormal spore was binucleate, receiving an $\mathrm{mR}-n u c l e u s$ in addition to its original Mr-nucleus ; Single-spore Culture 230-26 thus became doubly heterozygous.

It is worthy of note in this connection that, as a matter of principle, if an ascus yields a dicratic segregation-i.e. either $2 M R: 2 \mathrm{mr}$ or $2 M r: 2 m R$ and a second nucleus arising from an extra mitosis is incorporated in a spore, then two possibilities may occur : (I) the extra nucleus is of the same genetic constitution as the original nucleus whereby the binucleate condition of the spore could not be detected, or (2) the extra nucleus is genetically different from the original spore nucleus, whereby the subsequent diploid culture is necessarily doubly heterozygous-either $M R+m r$ or $M r+m R$. In tetracratic asci 
$(M R: M r: m R: m r)$, however, singly heterozygous single-spore cultures may arise from binucleate spores.

We investigated, in the same way as described above, the singlespore cultures of 6 of the 12 deviating asci listed in table 1 . In 4 of these asci it was found that one of the four single-spore cultures was doubly heterozygous. In the fifth ascus one of the four single-spore cultures was singly heterozygous $(M m R R)$, and in agreement with the above, this ascus gave a tetracratic segregation, the three spores which gave rise to the homozygous cultures having the formulas $M R, M r$, and $m R$. The sixth and final ascus we investigated yielded 4 homozygous cultures, $M M R R, M M r r, m m R R$, and $m m R R$, but it is obvious that also this case should theoretically be expected to occur. If such a deviating tetracratic ascus becomes 8-nucleate and 4-spored, the subsequent diploid single-spore cultures can naturally have arisen through diploidisation of the 4 haploid spores, $M R, M r, m R$, and $m R$. Here, the two $m r$-nuclei are not incorporated in any of the four spores.

Additional investigations, which will not be discussed in detail here, have shown that recessive mr-spores germinate more poorly than $M R$-, $M r$-, and $m R$-spores, and it is possible that $m r-n u c l e i$ cannot satisfactorily compete with the other nuclear types for the available cytoplasm. Analyses of tetrads in which all 4 spores germinate have disclosed the fact that $m r$-spores germinate poorly, for the dicratic ascus type $2 M R: 2 \mathrm{mr}$ often occurred with a lower frequency than the dicratic ascus type $2 M r: 2 m R$, even though these two types, in the absence of linkage, should occur with equal frequencies.

\section{GENETIC INVESTIGATIONS OF LINDEGREN'S HAPLOID YEASTS}

Professor Carl Lindegren, Southern Illinois University, Carbondale, Illinois, kindly supplied us with two haploid cultures from his collection, one of which could ferment maltose while the other could not. These haploid cultures were of opposite mating types and were designated as

$$
\begin{gathered}
1428-\alpha m a \\
15517-a M A
\end{gathered}
$$

since Lindegren does not, as we, designate the allelomorphs for maltose fermentation with $M$ and $m$, but with $M A$ and $m a$.

Since it is primarily Lindegren who has obtained so many tetrads giving deviating segregation ratios, which he would explain on the basis of gene conversion, this material was of special interest. It is worthy of note in this connection that Lindegren's two cultures lack the $D$ gene and therefore ascospores isolated from them normally give rise to haploid single-spore cultures. It was therefore a question whether our explanation of the deviating tetrads involving extra mitoses and binucleate spores was also valid for Lindegren's material.

The question can be definitely answered in the affirmative.

We produced a hybrid mixture between cultures 1428 and $155^{17} 7$ having the formula $\alpha a M A m a$, and we examined the segregation 
ratios of 34 asci with the result that 3 I segregated normally, $2 M A: 2 \mathrm{ma}$, while 3 asci gave a $3 M A$ : I ma segregation. We could thus confirm Lindegren's observations of irregular segregation in his material, but the explanation of this phenomenon has nothing in common with gene conversion, for in all 3 deviating asci we found that one of the three dominant $M A$ types was diploid and heterozygous while the three remaining cultures in the tetrad were haploid. These three irregular tetrads had the following formulas :

\section{MAma $\quad M A \quad M A \quad m a$}

As expected, the three haploid cultures were unable to sporulate, while the fourth culture produced spores.

Three asci from the diploid culture of the first irregular tetrad were analysed, and 2 segregated out as $2 M A: 2 m a$ and $\mathrm{I}$ as I $M A: 3 \mathrm{ma}$.

Five asci from the diploid culture of the second irregular tetrad were analysed, and 4 segregated out as $2 M A: 2 m a$ and $\mathrm{I}$ as $3 M A:$ I $m a$.

Two asci from the diploid culture of the third irregular tetrad were analysed, and both segregated out as $2 M A: 2 \mathrm{ma}$.

Thus two out of the ten asci we analysed showed again abnormal segregation, and there is no doubt that Lindegren's material, just as ours in the present investigation, possesses an inherited tendency toward extra mitoses in the ascus as well as toward the formation of binucleate spores.

In this connection the researches of Mundkur (1950) are of interest, since he also worked with Lindegren's material. As previously mentioned, Mundkur was correct in denying the possibility that our explanation of irregular segregation involving supernumerary mitoses could be employed for his results. It will be seen, however, that the fact that spores may sometimes be binucleate can account for irregular segregations, which Mundkur (as well as Lindegren) would explain on the basis of gene conversion.

Let us take as an example the tetrad mentioned by Mundkur in I950 which yielded abnormal segregation ratios. The spores of this tetrad had the following formulas, in spite of the fact that the hybrid from which they arose was heterozygous with respect to all genes investigated :

$\begin{array}{llllllll}\text { M 532 } & \alpha & G & M E & M G & M A & p n & \text { in } \\ \text { M 533 } & \alpha & G & M E & M G & m a & p n & \text { in } \\ \text { M 534 } & a & g & M E & M G & M A & P \mathcal{N} & I \mathcal{N} \\ \text { M 535 } & a & g & m e & M G & M A & P \mathcal{N} & I \mathcal{N}\end{array}$

(We have purposely omitted the gene pair ad $(P)-A D(W)$ because it behaves in a special way; the adenine-requiring red type, ad $(P)$, very often " mutates" to a white phenotype, ad $(W)$, which is methionine-dependent.) 
It can be seen that from this tetrad there have been segregated out $3 M E: 1 \mathrm{me}, 4 M G: 0 \mathrm{mg}$, and $3 M A: 1 \mathrm{ma}$. These deviating ratios can be reasonably explained in the following way.

Originally, the 4 primary nuclei in the ascus were :

$\begin{array}{llllllll}1: & \alpha & G & M E & M G & M A & p n & \text { in } \\ 2: & \alpha & G & m e & m g & m a & p n & \text { in } \\ 3: & a & g & M E & m g & M A & P \mathcal{N} & I \mathcal{N} \\ 4: & a & g & m e & M G & m a & P \mathcal{N} & I N\end{array}$

This constitutes a perfectly regular $2: 2$ segregation of all genes. But nucleus 1 and 4 (and possibly 2 and 3 as well) each undergo an extra mitotic division, and in two of the subsequent spores there are incorporated two nuclei so that the final tetrad consists of the following spores $(A, B, C, D)$ :

\begin{tabular}{|c|c|c|c|c|c|c|c|}
\hline $\begin{array}{l}\text { Spore A with nucleus I : } \\
\text { Spore } B \text { with nuclei } 1+2 \text { : } \\
\text { Spore } C \text { with nuclei } 3+4 \text { : } \\
\text { Spore } D \text { with nucleus } 4:\end{array}$ & $\begin{array}{l}\alpha \\
\alpha \alpha \\
a a \\
a\end{array}$ & $\begin{array}{l}G \\
G G \\
g g\end{array}$ & $\begin{array}{l}M E \\
M E m e \\
\text { MEme } \\
\text { me }\end{array}$ & $\begin{array}{l}M G \\
M G m g \\
M G m g \\
M G\end{array}$ & $\begin{array}{l}M A \\
\text { MAma } \\
\text { MAma } \\
m a\end{array}$ & $\begin{array}{l}p n \\
p n p n \\
P \mathcal{N} P \mathcal{N} \\
P \mathcal{N}\end{array}$ & $\begin{array}{l}\text { in } \\
\text { inin } \\
I N I N \\
I N\end{array}$ \\
\hline
\end{tabular}

Thus one will find by analysis the occurrence of $3 M E:$ I $m e$, $4 M G$ : o $m g$, and $3 M A:$ I $m a$, while all the rest of the gene pairs segregate normally. This is exactly what Mundkur found, although he apparently did not determine the haploidy or diploidy of the 4 single-spore cultures arising from this irregular ascus. It is not known how $2 \alpha$-nuclei or 2 a-nuclei-i.e. 2 nuclei belonging to the same mating type-will behave at spore germination nor how the subsequent single-spore cultures will develop. If they fuse, it is possible that the culture, in spite of its diploid nature, will be able to invoke a mating response in a culture of the opposite mating type. One may even imagine a diploid $\alpha \alpha$-type producing a mating reaction both with a haploid $a$-type and with a diploid aa-type. If, on the other hand, the nuclei do not fuse, one can expect the development of a chimerical culture-i.e. one composed of two haploids of the same mating type. When such a chimera is analysed in toto, it is naturally the dominant genes that will express themselves, just as in a hybrid. Giant colonies should disclose whether a true chimera were involved, but it is not known whether Lindegren and Mundkur used giant colonies as controls.

The single tetraploid ascus of Roman, Hawthorne, and Douglas (195I) and the published results of Roman and Sands (1953) should be mentioned in this connection, since it is possible to explain these results on the assumption that binucleate spores occurred in their material, which was originally obtained from Lindegren. The authors are fully aware of this possibility, and offer this as an explanation of their results, especially with regard to diploid mating types of the formula $\alpha \alpha$ and $a a$. However, neither analyses of $3:$ I segregations nor cytological studies were attempted.

It would seem that the occurrence of binucleate spores would be a more probable explanation than the regular segregation of an 
autotetraploid ascus nucleus which seems to be preferred by these authors.

\section{CYTOLOGICAL VERIFICATION OF THE OCCURRENCE OF SUPERNUMERARY MITOSES AND BINUCLEATE SPORES}

Even though much disagreement prevails among yeast cytologists, no difficulty is involved in obtaining stained preparations in which the number of nuclei can be observed in vegetative cells, asci during sporulation, and in the spores themselves. Lietz's cytological investigations (I95I) seem to us to be the most satisfactory so far, and, in general, we can confirm his observations except for the fact that in our material we were unable to distinguish the chromosomes so clearly as Lietz's drawings indicate he was able to do.

We are compelled to mention the cytological studies of Subramaniam and his colleagues (Subramaniam, I948, I953; Rao and Subramaniam, I953; and Thiagarajan and Subramaniam, I954). As far as we can tell, their so-called "chromosomes" may at times be identical with nuclei, but apparently by employing an unsatisfactory technique (e.g. osmium vapour fixation), these investigators have often obtained other stainable bodies in their preparations-bodies which show a typical "Spiegelfärbung" or other evidences of poor technique. Subramaniam's contentions ( $I$ ) that a fermenting cell is cytologically abnormal and incapable of survival, (2) that one should study the cytology of the yeast cell only from aerated cultures, and (3) that the transition from diplophase to haplophase has no connection with spore formation since the doubling and halving of the chromosome set is said to occur in the vegetative phase of the life cycle-these bear witness to a lack of understanding of the biology of the yeast cell. In addition, since Subramaniam and his colleagues have not undertaken a single genetic analysis of a yeast culture, nor have they undertaken any cytological investigations of the pre-sporulating phase of the life cycle, it is not surprising that work from their hands has become so misleading that they are not in agreement with any other investigators.

Excellent staining of chromatin material is obtained by fixation with Zenker's fluid followed by hydrolysis and staining with Feulgen or the still better Azure (DeLamater, I95I), but it is obvious that when the nucleus of such a stained yeast cell in the resting stage is ca. $0.5 \mu$ in diameter there is not much hope of being able to observe the chromosomes in detail under the ordinary light microscope.

We found Zenker's fluid to be the best fixative, and we employed I-day-old streak cultures as well as aerated cultures in a liquid medium.

Plate I, fig. I shows two nuclei each with an apparent doubleness (anaphase) in two haploid cells. Fig. 2 shows a haploid cell with its nucleus in the telophase ; a yet anucleate bud has been formed. Fig. 3 shows a diploid cell with a bud, both of which are uninucleate. Fig. 4 shows a young binucleate ascus, and a young 4-nucleate ascus 
is seen in fig. 5. In fig. 6 is shown an ascus with 4 uninucleate spores. Fig. 7 shows an ascus with 3 uninucleate spores; the fourth nucleus is seen lying free in the ascus. All of these stages are normal and found in large numbers in our preparations.

Figs. 8 and 9 are photographs of the same ascus in different foci. Both figures show clearly a binucleate spore, the ascus itself containing, as the result of extra mitoses, two binucleate spores. Also in fig. Io is seen a binucleate spore in an ascus. Figs. II and 12 show in different optical levels a non-synchronous nuclear division in a young ascus ; one nucleus is dividing and is in the telophase while the others are in the resting stage. Fig. I3 shows an 8-nucleate ascus, in which 5 nuclei are seen in focus. Finally, in figs. I4 and 15 may be seen in two optical levels 4 nuclei dividing synchronously; the 8 daughter nuclei are arranged in 4 pairs, one of which is not clearly in focus.

It is thus evident that there is complete agreement between the cytalogical observations and the genetic demonstration of the occasional occurrence in the ascus of supernumerary mitoses and the subsequent formation of binucleate spores.

\section{SUMMARY}

In the tetrad analysis of yeast fungi (Saccharomyces) there are occasionally found deviations from the theoretically expected simple mendelian $2: 2$ segregation ratios in the asci of monohybrids.

It has been claimed (Lindegren, Mundkur) that these deviating tetrads constitute proof of the correctness of Winkler's conversion theory, but the deviating tetrads investigated here did not arise by gene conversion. Neither are they the result of polyploidy, cytoplasmic inheritance, nor mutation. They are formed as the result of the occurrence of supernumerary mitoses within the ascus, resulting in 5-8 nucleate asci which are capable of producing binucleate spores.

Binucleate spores usually yield diploid heterozygous single-spore cultures. When a 3 dominant : I recessive type is segregated out in an ascus instead of $2 d: 2 r$, one of the 3 dominants is usually heterozygous.

It is shown also in Lindegren's yeast material, which normally gives rise to haploid single-spore cultures (our material diploidizes automatically owing to the presence of the $D$ gene) that when a $3 d$ : I $r$ segregation occurs, one of the three dominant types is always diploid and usually heterozygous ; this is due to the incorporation of 2 nuclei in the spore in question.

The deviating tetrads found in multiple heterozygous hybrids (Mundkur) may also be explained on the basis of the occurrence of binucleate spores.

Cytological investigations have confirmed the occurrence of supernumerary mitoses and binucleate spores within the ascus. 


\section{REFERENCES}

DELAMATER, E. D. 1951. A staining and dehydrating procedure for the handling of microorganisms. Stain Techn., 26, 199-204.

EPHRUSSI, B. 1953. Nucleo-Cytoplasmic Relations in Micro-Organisms. Oxford University Press.

GILLILAND, R. B. 1949. A yeast hybrid heterozygotic in four fermentation characters. Compt. Rend. d. Lab. Carlsberg, Ser. Physiol., 24, 347-356.

IIETz, к. I951. Beitrag zur Hefecytologie. Arch.f. Mikrob., 16, 275-302.

LINDEGREN, c. c. 1949. The Teast Cell, its Genetics and Cytology. Educational Publishers, Inc., St Louis.

LINDEgRen, c. c. 1953. Gene conversion in Saccharomyces. Four. Genet., 51, 625-637. MUNDKUR, B. D. 1949. Evidence excluding mutations, polysomy, and polyploidy as possible causes of non-mendelian segregations in Saccharomyces. Ann. Missouri Bot. Gard., 26, 259-280.

MUNDKUR, B. D. I950. Irregular segregations in yeast hybrids. Current Sci., 19, $84-85$.

RAO, L. S., PRAhlada AND subramantam, M. K. 1953. Studies on the cytology of yeasts. VII. Nuclear phenomena in cells from 24-hour agar slants. Proc. Indian Acad. Sci., 37, 72-81.

ROMAN, H., HAWTHORNe, 7. C., AND DOUglas, H. C. I951. Polyploidy in yeasts and its bearing on the occurrence of irregular genetic ratios. Natl. Acad. Sci. Proc., 37, 79-84.

ROMAN, H., AND SANDS, S. M. 1953. Heterogeneity of clones of Saccharomyces derived from haploid ascospores. Ibid., 39, I 7 I-1 79 .

SUBRAmaniam, M. K. I 948 . Studies on the cytology of yeasts. IV. Endopolyploidy in yeasts. Proc. Natl. Inst. Sci. India, 14, 325-333.

subramaniam, M. K. I953. Abnormal mitoses in tetraploid yeasts. Proc. Indian Acad. Sci., 37, 27-32.

thiagarajan, T. R., AND subramantam, M. K. I954. Studies on the cytology of yeasts. IX. Cytology of cells from a two-day-old giant colony. Arch.f. Mikrob., $20,183-200$.

WINGE, ø., AND ROBERTS, c. 1948. Inheritance of enzymatic characters in yeasts, and the phenomenon of long-term adaptation. Compt. Rend. d. Lab. Carlsberg, Ser. Physiol., 24, 263-315.

WINGE, Ø., AND ROBERTS, C. 1949. A gene for diploidization in yeasts. Ibid., 24, $34 \mathrm{I}-34^{6}$.

WINGE, Ø., AND ROBERTs, c. I950a. Non-mendelian segregation from heterozygotic yeast asci. Nature, 165,157 .

WINGE, ø., AND ROBERTS, c. I950b. The polymeric genes for maltose fermentation in yeasts, and their mutability. Compt. Rend. d. Lab. Carlsberg, Ser. Physiol., $25,35-83$.

WINGE, Ø., AND ROBERTS, c. 1952. The relation between the polymeric genes for maltose, raffinose, and sucrose fermentation in yeasts. Ibid., 25, I4 1 -1 7 I.

WINKLER, H. 1930. Die Konversion der Gene. Gustav Fischer, Jena. 
Plate $I$
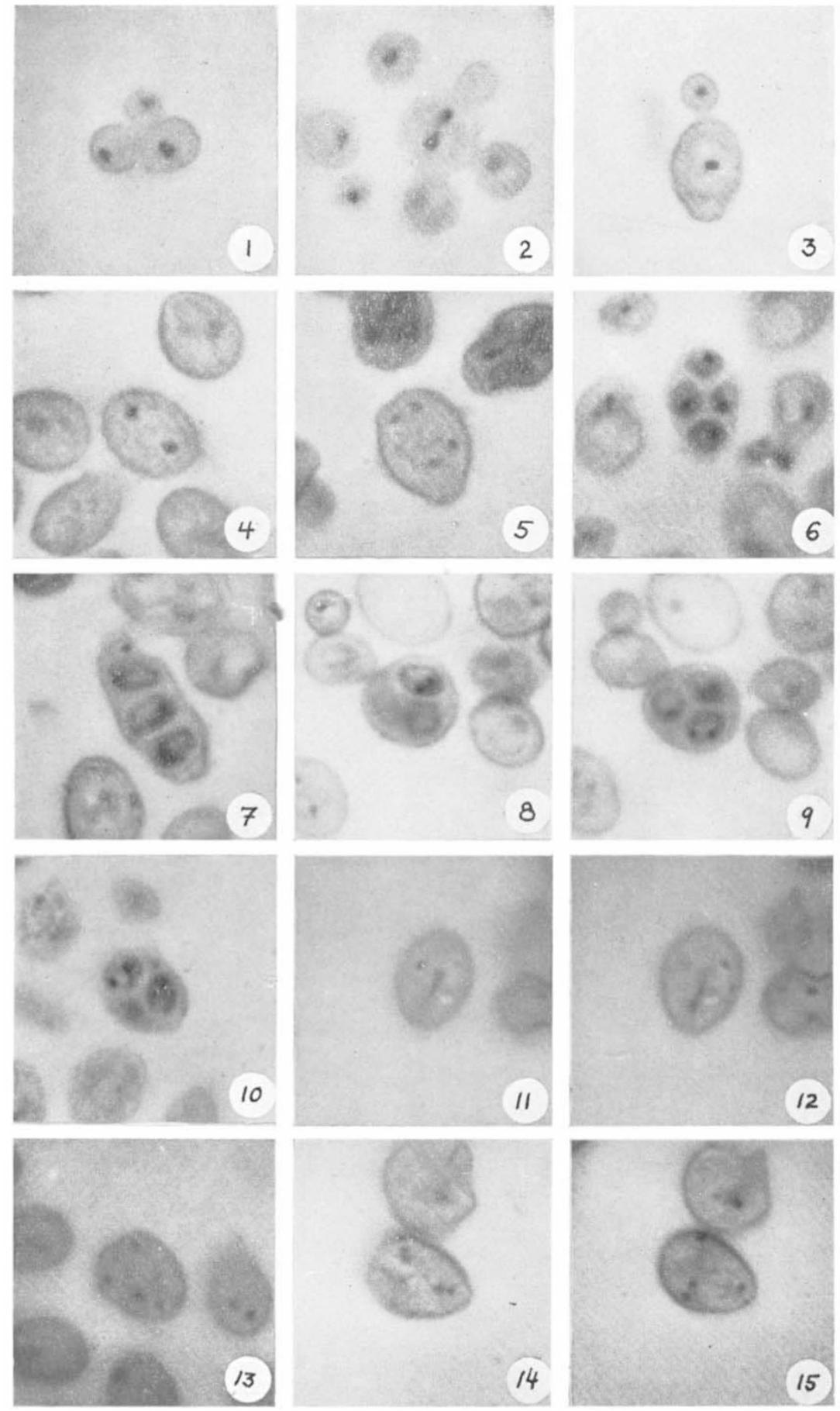

Cytological cvidence for the occurrence of supernumerary mitoses and binucleate spores in Saccharomyces (2700-2800 x). For explanation, see text, pages 302-303. 